\title{
Associative learning in ADHD: improved expression under methylphenidate
} \author{
Jackson $^{2}$

\footnotetext{
${ }^{1}$ School of Psychology, University of Nottingham University, Nottingham, UK;

${ }^{2}$ Division of Psychiatry, University of Nottingham, Nottingham, UK.

E-mail: *helen.cassaday@nottingham.ac.uk
}

Ebrahim Kantini ${ }^{1}$, Helen Joan Cassaday ${ }^{1 *}$, Martin Joseph Batty ${ }^{2}$, Chris Hollis ${ }^{2}$, Georgina Margaret

Received 12 May 2011; revised 7 June 2011; accepted 15 June 2011.

\section{ABSTRACT}

Attention Deficit/Hyperactivity Disorder (ADHD) is characterised by developmentally inappropriate levels of inattention, impulsivity and hyperactivity. As might be expected of a disorder in which inhibitory deficits form part of the diagnostic criteria, deficits in response inhibition in ADHD have been evidenced in a number of studies. To date, the tasks used in such studies have required participants to inhibit the learned stimulus-response associations that result in unwanted behavior. However, no research has examined the inhibition of stimulus-stimulus associations, formally 'conditioned inhibition'. The present study used video game style conditioned inhibition procedures, developed for children and adolescents with a clinical diagnosis of ADHD and suitable for typically developing matched controls. Two computer-based tasks ('Mission to Mars' and 'Weapon- $X$ ') required participants to predict the occurrence of an outcome based on the stimuli presented. We selected 12 male participants with ADHD on medication (methylphenidate), but without comorbid Tourette Syndrome, pervasive developmental disorder, learning disability or psychosis. This group showed overall normal inhibition of stimulus-stimulus associations, measured repeatedly over trials and with two task variants. There was no correlation between inhibitory learning and symptom severity ratings. However, participants with ADHD on higher dosages of methylphenidate, or longer duration of treatment with methylphenidate, showed improved ability to anticipate outcomes following the different stimulus presentations on non-inhibited versus inhibited trials. This effect was most clearly demonstrated on the Weapon-X task. Thus, methylphenidate dose-relatedly improved the expression of associative learning. This action may contribute to its therapeu- tic effects in improving cognitive function in ADHD.

Keywords: ADHD; Associative Learning; Conditioned Inhibition; Methylphenidate.

\section{INTRODUCTION}

Attention Deficit/Hyperactivity Disorder (ADHD) is a pervasive neurodevelopmental disorder characterised by developmentally inappropriate levels of inattention and hyperactivity/impulsivity [1-3]. The prevalence of ADHD has been variably estimated as high as $10 \%-18 \%[4,3]$, but is generally considered to be around 3 - 5\% [1].

As might be expected, a variety of experimental tasks, principally the 'stop signal' and 'Go/No-Go' task, have demonstrated deficits in response inhibition in children with ADHD [5-10]. From a learning theory perspective, these procedural tasks have the common feature that successful performance requires inhibition of prepotent stimulus-response (S-R) associations.

There have been few studies of stimulus-stimulus (S-S) learning in ADHD. Since the neural circuitries necessary for S-R and S-S associations are not equivalent, we cannot assume that the latter will also be abnormal in ADHD. In a study of blocking, Oades and Müller [11] found that the establishment of a prior S-S association was no bar to learning about an additional (redundant) stimulus in children with ADHD compared to matched controls. However, although the demonstration of blocking is based on S-S associations, the effect is not generally considered to be mediated by inhibition from the earlier association [12-14]. To our knowledge, no research has examined the inhibition of S-S associations (formally 'conditioned inhibition') in ADHD. In conditioned inhibition procedures, a conditioned stimulus (CS) is presented immediately prior to an unconditioned stimulus (UCS), except on those occasions when it is preceded by the conditioned inhibitor (CI). Thus, the CI 
comes to inhibit the CS-UCS association [15]. We have developed video game style conditioning procedures that demonstrate reliable conditioned inhibition and are suitable for younger participants [16,17]. In both tasks, summation tests measure the generalisation of the inhibitory properties of the CI to additional stimuli: a novel stimulus that does not appear at all in the training phase but is sufficiently similar to produce generalised responding $\left(\mathrm{S}_{\mathrm{g}}\right)$; and a transfer stimulus $\left(\mathrm{CS}_{\mathrm{t}}\right)$ that does not appear with the $\mathrm{CI}$ in the training phase. In both summation test variants, conditioned inhibition is demonstrated by a significant difference in the direction of UCS reinforced stimuli receiving higher expectancy ratings than non-reinforced stimuli presented with the pre-trained CI.

In the present study, these procedures were used to test children and adolescents with a clinical diagnosis of ADHD (corresponding to ICD-10 hyperkinetic disorder) and typically developing age and sex matched controls. Medication for ADHD has traditionally been with indirect dopamine agonists, of which methylphenidate is the most commonly used drug $[18,19]$. The participants with ADHD tested in the present study were all medicated with methylphenidate. In animal studies, conditioned inhibition is enhanced by treatment with amphetamine [20]. Thus, in addition to examining the prediction that conditioned inhibition would be impaired in ADHD, we examined the effect of dose and duration of treatment with methylphenidate.

\section{METHODS}

\subsection{Participants}

This study fully conformed to international guidelines on the ethical conduct of experimental work with human participants, as implemented in the UK. Ethical approval was obtained from the local research ethics committee and the R\&D Departments of the Nottinghamshire and Lincolnshire Partnership NHS Trusts (Derbyshire REC, 08/H0401/34, approved April 2008). After a complete description of the study, written informed consent and verbal assent was obtained from parents and children respectively.

\subsubsection{ADHD Group}

12 Children and adolescents with a clinical diagnosis of ADHD (12 males: mean age $=13$ years 11 months; range = 11 years 9 months -16 years 9 months) were recruited for the current study. The sample was part of a larger separate study, reported elsewhere [21,22]. Briefly, parents of children and adolescents with a diagnosis of ADHD were asked to attend an assessment session in which a battery of measures including the Development and Well Being Assessment (DAWBA; [23]), Strengths and Difficulties Questionnaire (SDQ; [24,25]), Conners' long form (CPRS-R: L; [26]) and Social and Communications Questionnaire (SCQ; [27]) were administered. Teacher completed versions of the DAWBA, SDQ and the CPRS-R: L were also available for each child. This, along with a review of medical records formed the basis for a clinical consensus diagnostic meeting, in which diagnosis was confirmed or overturned. Only children with an established positive response to methylphenidate and combined type ADHD were included. Any children with Tourette Syndrome (TS), pervasive developmental disorder, learning disability (defined as a full-scale intelligence quotient (FSIQ < 70), or psychosis were excluded. The demographic characteristics, details of medication with methylphenidate, and the symptom scores (CPRS-R: L) of the ADHD participants tested are summarised in Table 1.

\subsubsection{Control Group}

Of the 35 controls tested, 11 were matched for age ( \pm 6 months) and sex with the ADHD participants and included in the study (11 males: mean age $=13$ years 11 months; range $=11$ years 7 months -17 years 1 month). The control participants were screened for probable attentional problems using the Strengths and Difficulties Questionnaire [24], and asked whether they had any family members who had ADHD (although there was no formal assessment for psychiatric conditions) prior to testing. The matched control participants selected for inclusion in the study did not report difficulties indicative of any undiagnosed illness; similarly, none reported taking psychotropic medication for ADHD, or any other condition.

IQ scores were not available for all control participants. The IQ range of the participants with ADHD ( $n=$ 12) was 75 - 108 and 102 - 107 in the age-matched controls for whom IQ scores were available $(n=5)$. All of the participants with ADHD were on medication (Concerta - an extended-release preparation of methylphenidate) at the point of testing. Participant details are summarised in Table 1.

\subsection{Materials}

The task programmes were produced using E-prime (Psychology Software Tools Inc., Pittsburgh, USA) running on personal computers with 17" monitors, or on a 15” laptop computer when travel to the participant was required for testing. Participants' responses were made using a mouse.

\subsection{Procedure}

Behavioural procedures were identical to those used to test young participants with TS [16]. 
Table 1. Demographics, details of medication with methylphenidate, and symptom scores for the ADHD participants.

\begin{tabular}{|c|c|c|c|c|c|c|c|c|c|c|c|c|c|}
\hline & Participants & 1 & 2 & 3 & 4 & 5 & 6 & 7 & 8 & 9 & 10 & 11 & 12 \\
\hline \multirow{5}{*}{ 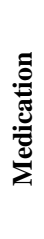 } & Age (months) & 201 & 190 & 161 & 141 & 144 & 167 & 169 & 190 & 145 & 142 & 153 & 194 \\
\hline & IQ (WASI) & 75 & 87 & 77 & 108 & 79 & 98 & 77 & 103 & 94 & 105 & 104 & 85 \\
\hline & Dosage (mg/kg) & 0.85 & 1.08 & 1.93 & 1.03 & 0.82 & 0.89 & 0.77 & 0.57 & 1.33 & 0.83 & 1.23 & 1.42 \\
\hline & Medication duration (months) & 63 & 41 & 51 & 5 & 19 & 22 & 18 & 92 & 11 & 54 & 59 & 27 \\
\hline & Medication duration corrected for age & 0.31 & 0.22 & 0.32 & 0.04 & 0.13 & 0.13 & 0.11 & 0.48 & 0.08 & 0.38 & 0.39 & 0.14 \\
\hline \multirow{16}{*}{ 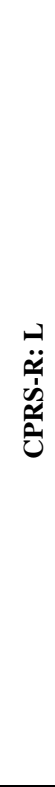 } & Oppositional & 80 & 84 & 90 & 87 & 85 & 90 & 89 & 54 & 83 & 85 & 75 & 90 \\
\hline & Cognitive Problems & 62 & 74 & 74 & 59 & 78 & 75 & 77 & 57 & 63 & 74 & 78 & 77 \\
\hline & Hyperactivity & 85 & 57 & 85 & 87 & 90 & 90 & 90 & 80 & 85 & 90 & 80 & 90 \\
\hline & Anxious & 54 & 44 & 61 & 45 & 79 & 90 & 47 & 51 & 63 & 45 & 63 & 68 \\
\hline & Perfectionism & 65 & 43 & 49 & 69 & 77 & 77 & 55 & 50 & 58 & 41 & 69 & 52 \\
\hline & Social Problems & 72 & 60 & 73 & 69 & 65 & 90 & 81 & 90 & 45 & 90 & 77 & 80 \\
\hline & Psychosomatic & 61 & 61 & 42 & 63 & 88 & 90 & 90 & 90 & 58 & 48 & 83 & 58 \\
\hline & ADHD Index & 70 & 77 & 77 & 73 & 77 & 83 & 79 & 60 & 75 & 82 & 76 & 75 \\
\hline & Global Restless Score & 63 & 70 & 86 & 77 & 82 & 90 & 86 & 70 & 80 & 86 & 75 & 81 \\
\hline & Emotional Lability & 90 & 73 & 83 & 78 & 72 & 90 & 83 & 61 & 72 & 72 & 83 & 79 \\
\hline & Global Total & 72 & 72 & 88 & 80 & 81 & 90 & 88 & 69 & 80 & 84 & 80 & 83 \\
\hline & DSM Inattentive & 69 & 76 & 77 & 65 & 80 & 81 & 71 & 58 & 65 & 80 & 77 & 78 \\
\hline & DSM Hyperactive & 86 & 63 & 81 & 87 & 90 & 90 & 87 & 81 & 81 & 90 & 81 & 90 \\
\hline & DSM Total & 78 & 73 & 81 & 77 & 88 & 90 & 80 & 69 & 74 & 90 & 81 & 89 \\
\hline & DSM Inattentive Count & 8 & 9 & 9 & 7 & 9 & 8 & 8 & 2 & 6 & 9 & 9 & 9 \\
\hline & DSM Hyperactivity Count & 6 & 2 & 7 & 9 & 9 & 9 & 9 & 6 & 7 & 9 & 8 & 8 \\
\hline
\end{tabular}

Legend: ADHD = Attention Deficit/Hyperactivity Disorder; mg/kg = milligrams medication per kilogram body weight dosage per day; CPRS-R: L = Conners' Parent Rating Scale-Revised: Long; WASI: Wechsler Abbreviated Scale of Intelligence. With the exception of the bottom two rows, which are counts (in the range 0-9), the CPRS-R: L ratings are $\mathrm{T}$ scores (mean $=50, \mathrm{SD}=10$ ).

\subsubsection{Conditioned Inhibition Task 1: 'Mission to Mars'}

The task scenario was based on a hypothetical mission to Mars and has been described in full elsewhere $[16,17]$.Participants were informed that they were to play the role of a commander of a fleet of starships travelling on an exploratory mission to Mars. However, trouble arises as, during the course of this mission, spaceships in the fleet keep mysteriously exploding.

The training phase consisted of 45 learning trials of the types shown in Table 2. During the training phase there were no explicit learning instructions; participants were simply asked to carefully count the number of surviving rockets.

There were 9 presentations of 5 trial types, which used different CSs presented with or without the CI: $\mathrm{CS}_{\mathrm{a}}+$, [CI, $\mathrm{CS}_{\mathrm{a}}$ ] -, $\mathrm{CS}_{\mathrm{b}}+$, [CI, $\mathrm{CS}_{\mathrm{b}}$ ] -, and $\mathrm{CS}_{\mathrm{t}}+$, where “+” signified reinforcement (i.e. rocket UCS presentation), and “-” signified non-reinforcement (i.e. no rocket UCS presentation, represented as an exploded rocket). The 5 trial types were presented in a random order. As the masking task, participants were required to keep track of the number of surviving spaceships, so that the associations to be learned were less obvious. On non-reinforced trials, a 1-second grey frame surrounding a blue screen was presented. This was the CI. On excitatory trials, there was a 1-second presentation of an empty blue screen (at the equivalent point in the stimulus sequence). Next, the CS (a large planet) was followed by 3 distractors (smaller planets) appearing and disappearing on the same screen for a combined total of 4 seconds, then the UCS (i.e. rocket presentation) on reinforced trials, or the absence of the UCS (i.e. exploded rocket presentation) on non-reinforced trials. The purpose of the distractor stimuli was to mask the absence of the CI cue and to control for external inhibition. The distractors were also intended to reduce the likelihood of direct associations between the inhibitor and the absence of the US, as this was represented as an alternative outcome. Participants were required to press any button on the mouse to continue on to the next presentation.

The testing phase immediately followed on from the 
Table 2. The stimulus combinations presented during the training and testing phase of the two tasks.

\begin{tabular}{cc}
\hline Training Phase & Testing Phase \\
\hline $\mathrm{CS}_{\mathrm{a}}$ & $\mathrm{CS}_{\mathrm{t}}{ }^{+}$ \\
{$\left[\mathrm{CI}, \mathrm{CS}_{\mathrm{a}}\right]-$} & {$\left[\mathrm{CI}, \mathrm{CS}_{\mathrm{t}}\right]-$} \\
$\mathrm{CS}_{\mathrm{b}}{ }^{+}$ & $\mathrm{S}_{\mathrm{g}}{ }^{+}$ \\
{$\left[\mathrm{CI}, \mathrm{CS}_{\mathrm{b}}\right]-$} & {$\left[\mathrm{CI}, \mathrm{S}_{\mathrm{g}}\right]-$} \\
$\mathrm{CS}_{\mathrm{t}}+$ & \\
\hline
\end{tabular}

Legend: A '+' indicates the presentation of the UCS (i.e. an intact rocket for the Mission to Mars and a picture of Wolverine for the Weapon X task). A '-' indicates the absence of the UCS (i.e. an exploded rocket for the Mission to Mars and a picture of Feral Logan for the Weapon X task).

training phase and consisted of 20 further trials. There were 5 presentations of 4 trial types: $\mathrm{S}_{\mathrm{g}}+,\left[\mathrm{CI}, \mathrm{S}_{\mathrm{g}}\right]-, \mathrm{CS}_{\mathrm{t}}{ }^{+}$, and $\left[\mathrm{CI}, \mathrm{CS}_{\mathrm{t}}\right]-$, in which $\mathrm{S}_{\mathrm{g}}$ was a generalised stimulus not previously introduced during the training phase (described above). The 4 trial types were pre- sented in a random order. The procedure for test trials was identical to that used in training, except that prior to the presentation of the US (or its absence), participants were presented with an on-screen rating, scaled 1 - 9. At this point, participants were required to estimate the likelihood of the spaceship surviving, with a rating of 9 to represent the highest likelihood of survival, and a rating of 1 to represent the lowest likelihood of survival. An intermediate rating of 5 represented uncertainty. Ratings were made by selecting the appropriate on screen box using the mouse. Figure 1 shows the stimuli used in the training and test phases of the experiment.

This was a summation test of conditioned inhibition. Participants' ratings provided a measure of the inhibitory properties of the CI using two kinds of test stimuli: (1) $\mathrm{S}_{\mathrm{g}}$ (a stimulus that was from the same category but had not been explicitly pre-trained); and (2) $\mathrm{CS}_{\mathrm{t}}$ (a stimulus familiar from training, but that had not previously been preceded by the $\mathrm{CI}$ ).

\subsubsection{Conditioned Inhibition Task 2: 'Weapon- $X$ '}

This task presented a scenario based on the Weapon-X comic book story and has been described in full elsewhere [16]. Participants were informed that they were to play the role of Professor Thorton, Director of the Weapon-X project, with the job to create the ultimate living weapon, using metallurgic skeletal bonding to convert Logan into Wolverine. Failure results in the feral mutation of Logan. Participants were further informed that in order to learn Thorton's secret, they were to carefully observe his work in order to work out the causes of success (Wolverine) versus failure (the feral mutation). Thus, they were explicitly instructed to try to discover the cause of the outcome.

As for the previous task, the training phase consisted of 45 learning trials, presented as 9 presentations of the 5

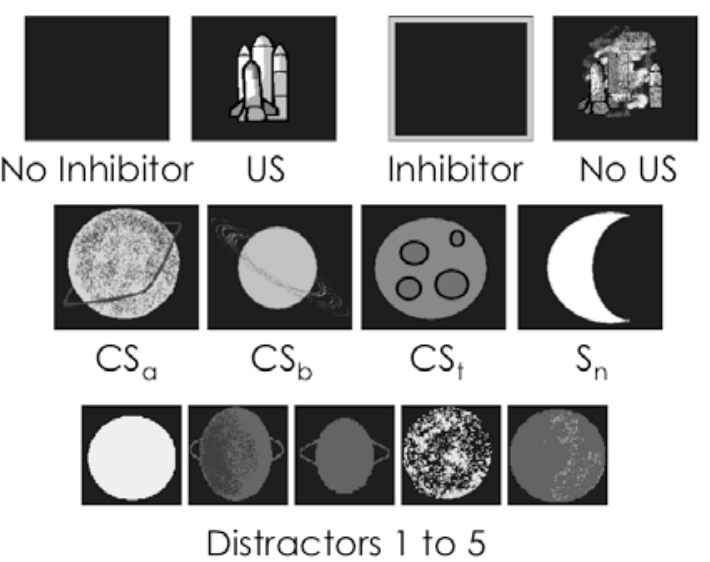

Figure 1. The screen shots used during the Mission to Mars task. Upper panel shows the blank screen presented in the absence of the inhibitor followed by the unconditioned stimulus (US) presentation and the alternative framed screen with a grey border used on inhibited trials followed by the absence of the desired US, depicted as an exploded rocket. The middle panel shows the alternative conditioned stimuli (CSs) including the inhibited transfer stimulus $\left(\mathrm{CS}_{\mathrm{t}}\right)$ and the generalised stimulus $\left(\mathrm{S}_{\mathrm{g}}\right)$. The bottom panel shows the distractor stimuli used to control for external inhibition.

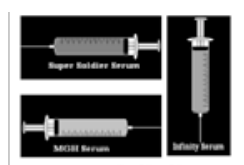

No Inhibitor

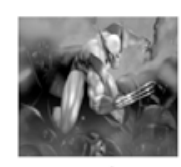

US

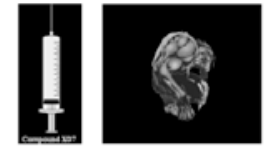

Inhibitor No US

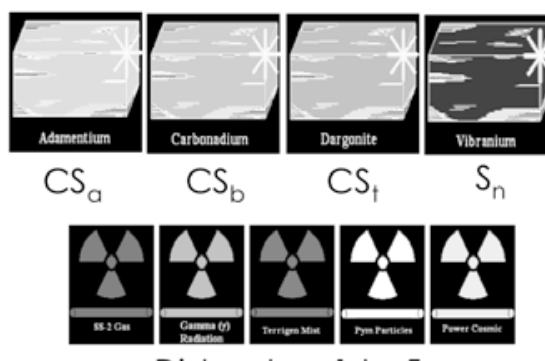

Distractors 1 to 5

Figure 2. The screen shots used during the Weapon-X task. Upper panel shows alternative syringe stimuli shown in the absence of the inhibitor followed by the unconditioned stimulus (US) presentation and the syringe presented on inhibited trials followed by the absence of the desired US, depicted as the unsuccessful transformation. The middle panel shows the alternative conditioned stimuli (CSs) including the inhibited transfer stimulus $\left(\mathrm{CS}_{\mathrm{t}}\right)$ and the generalised stimulus $\left(\mathrm{S}_{\mathrm{g}}\right)$. The bottom panel shows the distractor stimuli used to control for external inhibition.

trial types shown in Table 2. The stimuli were different in that in this task variant, participants were asked to observe a computer simulation of the Weapon- $X$ transformation. This consisted of 3 stimuli appearing simultaneously: the CI (represented by a yellow syringe) or its absence (when instead one of three alternative syringes 
was presented, see Figure 2 upper panel), the CS (a block of a certain fictitious alloy), and one of the five distractors (various types of radiation, see Figure 2 lower panel), for a total of 4 seconds on screen. The purpose of the alternative syringe stimuli was to mask the otherwise notable absence of the CI cue and to control for external inhibition (as above). The radiation distractors were to further reduce the likelihood of direct associations between the inhibitor and the absence of the US, as this was represented as an alternative outcome. These images were followed by a 1 second presentation of an image of Logan in the midst of the attempted transformation, then the presentation of the US (or its absence). The success or failure of the metallurgical bonding was represented by an image of Wolverine as the UCS, or a picture of feral Logan representing the absence of the UCS.

Participants were required to press any button on the mouse to continue. As above, the testing phase consisted of 20 further trials (as per Table 2) with the key generalised $\left(\mathrm{S}_{\mathrm{g}}\right)$ and transfer stimuli $\left(\mathrm{CS}_{\mathrm{t}}\right)$ for the summation test of conditioned inhibition. The procedure for test trials was identical to that used in training, except that prior to the presentation of the US or its absence, participants were presented with an onscreen rating on the scale 1-9, with 9 representing the highest likelihood of success. All choices were made by selecting the appropriate box on screen using the mouse. Figure 2 shows the stimuli used in the training and test phases of the experiment.

All participants were tested on both task variants, in a counterbalanced order, so practice effects could not account for any difference in performance on the two tasks.

\subsubsection{Design and Analysis}

Analysis of variance (ANOVA) was run in a mixed design with up to four within-subjects factors to assess conditioned inhibition by participants' summation test performance: inhibition (the presence or absence of the $\mathrm{CI}$ ); task (Mission to Mars versus Weapon-X); stimulus type (summation test with $\mathrm{S}_{\mathrm{g}}$ versus $\mathrm{CS}_{\mathrm{t}}$ ); test phase presentation (of which there were five levels). To improve focus, analyses were subsequently collapsed by stimulus type and test phase presentation where these factors did not interact with inhibition [28]. Diagnosis was the between subjects factor.

In order to investigate any possible effects of medication, median split analyses were conducted with respect to duration of medication (with correction for participant age) and the dosage of medication ( $\mathrm{mg} / \mathrm{kg}$ ). These were distinct parameters in that duration and dose of medication were not correlated (see below). Median split analysis was also used to examine the effect of symptom severity (measured using the CPRS-R: L) on the expres- sion of conditioned inhibition. The dependent variable to assess the expression of associative learning was the participants' expectancy score (for appearances of an intact rocket in task 1 or the successful transformation of Logan into Wolverine in task 2). Planned comparisons (two-tailed t-tests) were conducted to examine effects of a priori interest. In addition, effect sizes of likely interest are reported (Cohen's d).

A conditioned inhibition ratio was calculated by dividing the average expectancy score for non-inhibited stimulus presentations by the average expectancy score for inhibited stimulus presentations. Thus, conditioned inhibition is indicated by a ratio greater than one and the absence of conditioned inhibition by a ratio less than or equal to one. The interrelationship between the level of conditioned inhibition summarised by the ratio and symptom severity scores (measured by the CPRS-R: L) was explored by Pearson's $r$ correlation, 2-tailed. Where data were available (for all ADHD participants and 5 matched controls), the same analyses were repeated to examine summation test performance on each of the tasks in relation to IQ. Similarly, the effect of medication was further examined by correlational analysis, using duration of medication, duration of medication adjusted for age (months on medication divided by the age of the participant) and medication dosage (mg/kg).

\section{RESULTS}

Analysis with respect to the diagnostic groups (ADHD and matched controls), confirmed that there was a significant main effect of inhibition $\left(\mathrm{F}_{1,21}=24.782, p<\right.$ 0.001 ). However, there was no significant interaction between diagnostic group and inhibition $\left(F_{1,21}=0.763\right)$. Neither was there any interaction between task, inhibition and diagnostic group $\left(\mathrm{F}_{1,21}=0.029\right)$. Both the matched controls $\left(\mathrm{t}_{11}=3.624, p<0.005, d=2.16\right)$ and the ADHD group ( $\left.\mathrm{t}_{11}=3.374, p<0.01, d=1.94\right)$ demonstrated an overall effect of inhibition at the summation test (Figure 3). There were no significant interactions between inhibition or diagnostic group with respect to either stimulus, and/or presentation ( $\max \mathrm{F}_{1,21}=1.906, p$ $=0.182$ ). However, there was a marginal task by inhibition interaction $\left(\mathrm{F}_{1,21}=4.11, p=0.055\right)$ reflecting better performance in the Weapon- $X$ than in the Mission to Mars task.

The analysis of effects by medication was confined to the ADHD group alone. As expected, the effect of inhibition remained significant $\left(\mathrm{F}_{1,11}=11.384, p<0.01\right)$. Since there were again no significant interactions between inhibition and stimulus or presentation ( $\max F_{1,21}=1.388$ ), all further analyses were collapsed across the factors of stimulus and presentation. However, analyses were con 


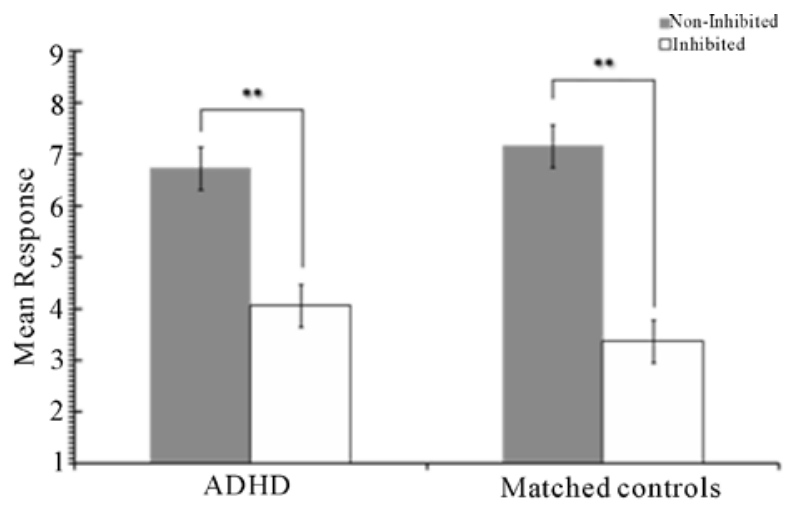

Figure 3. The overall effect of inhibition in the ADHD and matched control group. Mean Response refers to the participants' expectancy ratings (scaled 1 - 9) with a rating of 9 to represent the highest likelihood of the outcome (see text for further details). $* *=p<0.01$.

ducted separately by task because of the difference by task identified above. Figure 4 shows that participants who were below or equal to the median for medication dose, or duration of medication (with or without adjustment for age) did not show significant conditioned inhibition in either task variant $\left(\max t_{5}=1.955, d=0.99\right)$.In contrast, significant summation test discrimination was demonstrated in the Weapon- $X$ task variant by participants above the median dose $\left(\mathrm{t}_{5}=5.167, p<0.01, d=\right.$ 4.5), or above the median duration of medication (with or without adjustment for age, $\mathrm{t}_{5}=3.042, p<0.05, d=$ 2.62). Figure 4 shows that, in the Weapon- $X$ task, non-inhibited and inhibited ratings were respectively higher and lower above the median medication parameters. However, the only significant change was for the non-inhibited ratings in relation to dose $\left(\mathrm{t}_{10}=2.237, p<\right.$ $0.05, d=1.41)$. No other differences between the inhibited and non-inhibited ratings reached significance $\left(\max \mathrm{t}_{10}=1.341, d=0.85\right)$.

Although somewhat increased in those participants with longer duration of treatment, the improvements in summation test discrimination demonstrated above the median medication parameters were not significant in the Mission to Mars task variant $\left(\max t_{5}=1.671, d=1.4\right)$.

Again within the ADHD group, there was no interaction between symptom level (median split on the CPRS-R: L ratings) and inhibition or inhibition by task (max $\mathrm{F}=$ 0.666). Thus, high and low symptom participants showed equivalent summation test discrimination. Nevertheless, participants may have shown individual variation on the task in relation to symptom severity. To address the likely confound between medication status and ADHD symptom severity, ANCOVA was applied to the median split analyses for the ADHD group, using the ADHD index of the CPRS-R: L as covariate.

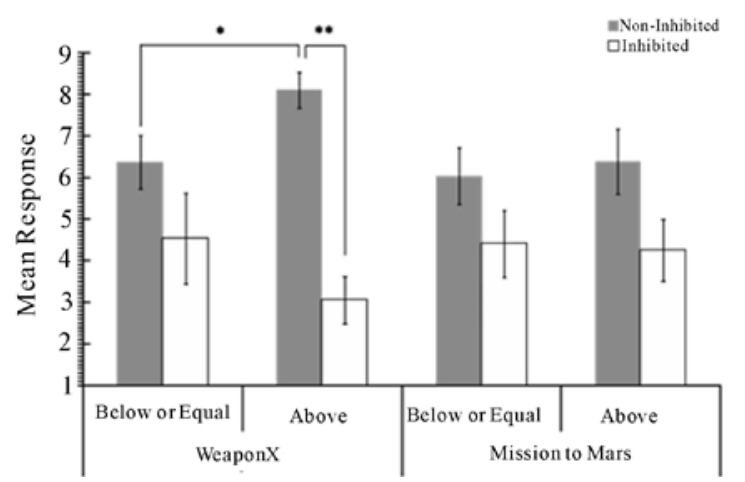

(a)

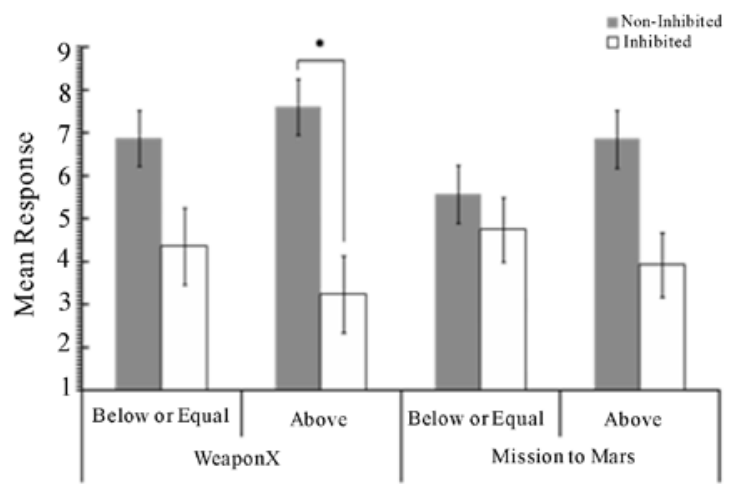

(b)

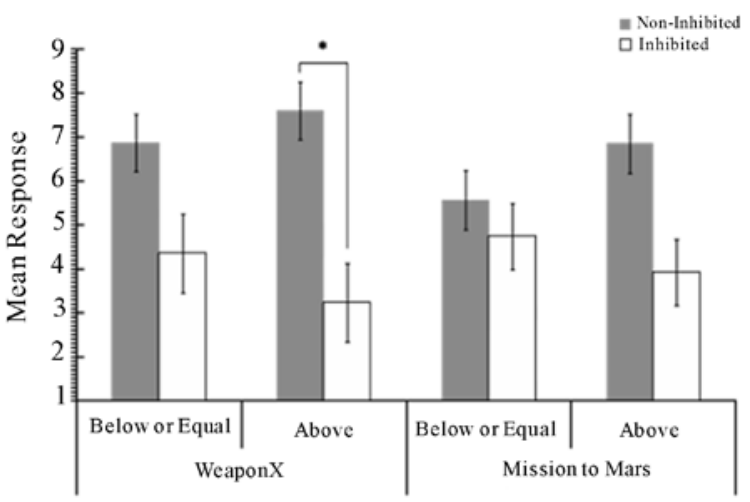

(c)

Figure 4. The effects of medication on summation test discrimination within the ADHD group. (a) ADHD group: differences by medication dosage; (b) ADHD group: differences by duration of medication; (c) ADHD group: differences by duration of medication adjusted for age. Effects of medication shown separately for the two task variants, with respect to (panel A) below or equal versus above median dosage [mg/kg], (panel B) below or equal versus above median duration of medication and (panel $\mathrm{C}$ ) below or equal versus above median duration of medication adjusted for age $(*=p<0.05$, ** $=p<$ $0.01, * * *=p<0.001$ ). Mean Response refers to the participants' expectancy ratings (scaled 1 - 9) with a rating of 9 to represent the highest likelihood of the outcome (see text for further details). 
There were no significant interactions between dose and inhibition ( $\max F_{1,9}=1.629$ ). However, a significant interaction between duration of medication and inhibition was found $\left(F_{1,9}=5.748, p<0.05\right)$. Inspection of the adjusted means shows that when symptom severity was taken into account, overall summation test discrimination was more pronounced in participants above the median duration of medication (mean inhibited ratings = 3.37, s.e.m. $=0.526$; mean non-inhibited ratings $=7.519$, s.e.m. $=0.405$ ) compared with summation test discrimination seen in participants below the median time on medication (mean inhibited ratings $=4.747$, s.e.m. $=$ 0.526, mean non-inhibited ratings $=5.922$, s.e.m. $=$ 0.405). Repeating the analysis with duration of medication adjusted for age yielded the same outcome. No other interactions were significant $(\max F<1)$.

Due to the design of the two tasks, no data were collected during the training phase of the procedure. However, in order to analyse whether participants demonstrated equivalent levels of learning at the end of the training phase, the ratings from the first presentation of the non-inhibited transfer stimulus during the test phase were analysed. Univariate ANOVA of the first presentation ratings demonstrated no significant difference between the ADHD group and the matched controls overall, for the Weapon-X task ( $\mathrm{F}=0.271)$, the Mission to Mars task $\left(\mathrm{F}_{1,21}=2.248\right)$, or over both tasks combined $(\mathrm{F}=$ 0.266).

Moreover, in order to test whether learning under methylphenidate had affected baseline excitatory learning, the analysis was repeated with medication dosage (median split) and duration of medication (median split) as the between subject variables.

Univariate ANOVA of the first presentation ratings demonstrated no significant difference between the two medication dosage groups for the Weapon- $\mathrm{X}$ task $(\mathrm{F}=$ $0.156)$, the Mission to Mars task ( $F=0.015$ ), or over both tasks combined $(\mathrm{F}=0.084)$. Likewise, there were no significant differences between the duration (high vs. low) of medication groups for the Weapon-X task $\left(\mathrm{F}_{1,10}=\right.$ 1.607), the Mission to Mars task ( $F=0.015)$, or over both tasks combined $(\mathrm{F}=0.236)$.

\subsection{Correlational Analyses}

Within the ADHD sample, there was no significant correlation between dose or duration of medication and performance on either task as summarised by the conditioned inhibition ratios ( $\max r_{12}=-0.408$ ). Dose and duration were distinct medication parameters in that there was no correlation between medication dosage and participants' time on medication $\left(r_{12}=-0.168\right)$. In addition, there was no correlation between medication dosage and participants' time on medication adjusted by age $\left(r_{12}\right.$
$=-0.115$ ), or between symptom severity measured by the CPRS-R: L (either overall or by its subscales; $\max r_{12}=$ -0.511 ), or between IQ and the summary scores provided by the conditioned inhibition ratios for either task (max $r_{12}=-0.549$ ). Similarly, the overall correlation between conditioned inhibition and IQ across both the ADHD and matched controls for whom IQ scores were available was also non-significant for both task variants (max $r_{17}=$ $-0.426)$.

\section{DISCUSSION}

Two variants of the summation test showed that inhibition was transferred to a CS that had not been previously presented with the $\mathrm{CI}$ during training $\left(\mathrm{CS}_{\mathrm{t}}\right)$ as well as to a novel stimulus from the same category to which excitatory responding was generalised $\left(S_{g}\right)$ [14]. Moreover, conditioned inhibition was tested using two task variants. The first (Mission to Mars) was a modified version of an established task, which used serial presentation of the CI followed by $\mathrm{CS}_{\mathrm{t}}$ or $\mathrm{S}_{\mathrm{g}}$ [17]. The second (Weapon-X) was a novel task, similarly designed to be engaging for younger participants, which used simultaneous presentation of the CI together with $\mathrm{CS}_{\mathrm{t}}$ or $\mathrm{S}_{\mathrm{g}}$. Since the originally developed task tested learning implicitly, the novel task variant used explicit learning instructions, to further confirm the generality of observed effects.

Conditioned inhibition was successfully demonstrated in all participants in the present study. Thus, young males with ADHD can successfully suppress S-S associations. However, there was variation within the ADHD group, in that participants on either a higher dosage or longer duration of treatment with methylphenidate showed improved performance and, consistent with some overall difference in inhibition by task, this effect was most clearly demonstrated in the Weapon-X variant. The difference took the form of improved summation test discrimination, but the improvement was not solely attributable to lower ratings given on inhibited stimulus presentations.

Summation test performance reflects the expression of prior learning. The course of acquisition was not directly assessed in the training phases of the tasks due to the implicit nature of the Mission to Mars task and the need to keep the tasks formally equivalent as far as possible. Analysis of first test trial responding to $\mathrm{CS}_{\mathrm{t}}$, which was used as the best estimate of (effects on) excitatory learning did not show any effect of diagnostic group or medication status. Although the profile of action on the summation test is consistent with generally improved associative learning under methylphenidate, the present results only allow the conclusion that the expression of prior learning was improved under methylphenidate. Moreover, there were differences by task in that the ac- 
centuated summation test performance only reached significance in the Weapon-X variant (see also below).

\subsection{Effects of Symptom Severity and IQ}

There was no detectable correlation between the CPRS-R: L scores of the ADHD participants (either overall or by its subscales) and the level of inhibition demonstrated in either task variant. This lack of correlation between symptom severity and conditioned inhibition could be due to a ceiling effect, given the relatively restricted range of scores in the patient sample who all met diagnostic criteria for ADHD, and/or the limited sample size. However, there was some indication in the data that the effect of medication was more than would be expected on the basis of symptom severity: when symptom severity was taken into account statistically (by analysis of covariance), there was an interaction between duration (but not dose) of medication and inhibition - in this case overall rather than separately by task. This result shows that, when symptom severity was taken into account, the summation test discrimination was improved overall in participants treated with methylphenidate over a longer time frame.

While a number of the participants with ADHD had a lower than average IQ, there was no correlation between IQ and the level of inhibition demonstrated, either within the ADHD sample, or including the matched controls where data were available. Conventional tests of inhibition used with ADHD participants (the stop signal, the Go/No-Go and a modified version of the Stroop task), similarly showed a deficit in relation to ADHD, but no association with IQ [29]. In the present study, it was not possible to match controls on the basis of IQ, as IQ scores were not available for all participants. However, there are arguments against matching on the basis of IQ in that disorders such as ADHD are a likely cause of depressed IQ: the matching fallacy whereby participants may be 'overmatched' on variables which are not independent of the disorder in question [8].

\subsection{Differences by Task}

Although conditioned inhibition was overall demonstrated in both task variants, performance was generally better on the Weapon- $X$ variant. This difference may arise because the storyline of the latter task was intrinsically more engaging for the young males tested in the present study, or because the use of explicit learning instructions influences task solution [30,31]. Alternatively, some advantage on the Weapon- $\mathrm{X}$ task may have been conferred by the simultaneous rather than serial presentation. Serial or 'occasion setting' versions of conditioned inhibition tasks provide a more direct analogue of response learning tasks, where the required association is qualified by discriminative stimuli (e.g., Go/No-Go). However, they may be generally more difficult. Animal studies have similarly indicated that the effectiveness of a stimulus as an inhibitor can depend on its temporal positioning with respect to potential excitors, and specifically that simultaneous rather than serial training more reliably results in conditioned inhibition as measured by summation test [32,33].

\subsection{Differences by Medication}

In participants with TS, medication with clonidine was found to impair the expression of conditioned inhibition, measured using identical procedures [16]. In the current study, the entire sample of ADHD participants was medicated. Moreover, the level of conditioned inhibition was not equivalent across the two tasks, and the ADHD group did not show significant conditioned inhibition on the Mission to Mars task. Accordingly, within the ADHD sample, the effects of medication were examined separately by task. Correlational analyses did not suggest any linear relationship between the level of conditioned inhibition and medication dose or duration in either task variant. However, median split analyses to divide the ADHD group into low v. high dose and short v. long duration of treatment showed that (according to either of these medication parameters) treatment with methylphenidate tended to improve expression of the conditioned inhibition discrimination measured at the summation test, significantly so for the Weapon- $\mathrm{X}$ task. This difference by conditioned inhibition variant appears related to task difficulty, in that the Weapon-X task supported a stronger conditioned inhibition effect (presumably in consequence of its explicit learning instructions and simultaneous rather than serial presentation of the CI in relation to $\mathrm{CS}_{\mathrm{t}}$ and $\mathrm{S}_{\mathrm{g}}$ ). The Weapon- $\mathrm{X}$ task also supported better performance in the unmedicated controls.

In animal studies too, methylphenidate has been found to increase simple associative learning [34,35]. The neural substrates of specifically inhibitory learning have been little investigated to date, but the effect of amphetamine is to enhance conditioned inhibition [20]. This finding is similarly consistent with the possibility of differences in relation to ADHD and its medication with indirect dopamine agonists.

\subsection{Conclusions}

The participants with ADHD tested in the present study were all medicated with methylphenidate; treatment was thus confounded with diagnosis. Moreover, differences may have been masked by ceiling effects, since participants were selected on the basis of high scores on the CPRS-R: L scale. Thus, the relationship between symp- 
tom severity and performance in the conditioned inhibition tasks should be further investigated in a larger, more heterogeneous sample. Relatedly, our procedures were not suitable for repeat testing with the same task and the effects of medication point to the need to test the hypothesis that the inhibition of S-S associations should be impaired in ADHD when medication is withdrawn. However, effects on the expression of associative learning in relation to medication in ADHD are nonetheless of interest. Participants below the median dose or duration of treatment with methylphenidate showed no discrimination on the summation test, whereas the summation test discrimination was significant above these medians with a small to medium effect size (ds of 4.5 and 2.62, respectively).

Methylphenidate has previously been reported to improve the capacity to inhibit ongoing and prepotent responses in conventional tests of response inhibition, including the Stroop [36] and variants of the stop signal task [37]. Although the present study demonstrated improved performance of the key summation test discrimination in a conditioned inhibition procedure, this result cannot be taken to reflect enhanced inhibition of S-S associations under methylphenidate because the improvement in discrimination performance had two components. Moreover, only the change in non-inhibited ratings reached significance (for the Weapon-X task, when the sample was split on the dosage parameter). Thus, although there was no evidence of drug effects on excitatory learning, as measured by trial 1 responding to the $\mathrm{CS}_{\mathrm{t}}$, neither was there any evidence that the improved summation test discrimination arose because of a change in inhibitory as distinct from excitatory learning. We therefore conclude that methylphenidate improved the expression of associative learning in general, and suggest that this action may contribute to its therapeutic effects in improving cognitive function in ADHD.

\section{ACKNOWLEDGEMENTS}

Ebrahim Kantini was supported by an Overseas Research Student Award, held at the School of Psychology. We thank Jane Fowlie for her help with data collection.

Declaration of competing interests: The authors declare that they have no competing interests.

\section{REFERENCES}

[1] American Psychiatric Association (2000) Diagnostic and statistical manual of mental disorders, fourth edition, text revision (DSM-IV-TR). American Psychiatric Association, Washington.

[2] Barkley, R.A. (1998) Attention-deficit/hyperactivity disorder: A handbook for diagnosis and treatment. 2nd Edition, Guilford Press, New York, 75-143.
[3] Faraone, S.V., Sergeant, J., Gillberg, C. and Biederman, J. (2003) The worldwide prevalence of ADHD: Is it an American condition? World Psychiatry, 2, 104-113.

[4] Kytja, K.S. and Voeller, M.D. (2004) Attention-Deficit hyperactivity disorder (ADHD). Journal of Child $\mathrm{Neu}$ rology, 19, 798-814.

[5] Iaboni, F., Douglas, V.I. and Baker, A.G. (1995) Effects of reward and response costs on inhibition in ADHD children. Journal of Abnormal Psychology, 104, 232-240. doi:10.1037/0021-843X.104.1.232

[6] Schachar, R. and Logan, G.D. (1990) Impulsivity and inhibitory control in normal development and childhood psychopathology. Developmental Psychology, 26, 710720. doi:10.1037/0012-1649.26.5.710

[7] Schachar, R., Mota, V.L., Logan, G.D., Tannock, R. and Klim, P. (2000) Confirmation of an inhibitory control deficit in attention-deficit/hyperactivity disorder. Journal of Abnormal Child Psychology, 28, 227-235. doi:10.1023/A:1005140103162

[8] Seidman, L.J., Biederman, J., Faraone, S.V., Wever, W. and Ouellette, C. (1997) Toward defining a neuropsychology of attention deficit-hyperactivity disorder: Performance of children and adolescents from a large clinically referred sample. Journal of Consulting and Clinical Psychology, 65, 150-160. doi:10.1037/0022-006X.65.1.150

[9] Trommer, B.L., Hoeppner, J.-A.B., Lorber, R. and Armstrong, K.J. (1988) The Go-No-Go paradigm in attention deficit disorder. Annals of Neurology, 24, 610-614. doi:10.1002/ana.410240504

[10] Young, S., Bramham, J., Tyson, C. and Morris, R. (2006) Inhibitory dysfunction on the Stroop in adults diagnosed with attention deficit hyperactivity disorder. Personality and Individual Differences, 41, 1377-1384. doi:10.1016/j.paid.2006.01.010

[11] Oades, R.D. and Müller, B. (1997) The development of conditioned blocking and monoamine metabolism in children with attention-deficit-hyperactivity disorder or complex tics and healthy controls: An exploratory analysis. Behavioural Brain Research, 88, 95-102. doi:10.1016/S0166-4328(97)02306-1

[12] Rescorla, R.A. and Wagner, A.R. (1972) A theory of Pavlovian conditioning: Variations in the effectiveness of reinforcement and nonreinforcement. In: Black, A.H., Prokasy, W.F., Eds., Classical Conditioning II: Current Research and Theory, Appleton-Century-Crofts, New York, 64-99.

[13] Escobar, M., Oberling, P. and Miller, R.R. (2002) Associative deficit accounts of disrupted latent inhibition and blocking in schizophrenia. Neuroscience \& Biobehavioral Reviews, 26, 203-216. doi:10.1016/S0149-7634(01)00067-7

[14] Rescorla, R.A. (1969) Pavlovian conditioned inhibition. Psychological Bulletin, 72, 77-94. doi:10.1037/h0027760

[15] Miller, R.R. and Matzel, L.D. (1988) The comparator hypothesis: A response rule for the expression of associations. In: Gordon, H. B. Ed., Psychology of Learning and Motivation, Academic Press, Waltham, 51-92.

[16] Kantini, E., Cassaday, H.J., Hollis, C.P. and Jackson, G.M. (2011) The normal inhibition of associations is impaired by clonidine in Tourette syndrome. Journal of the Canadian Academy of Child and Adolescent Psychiatry, 20, 
96-106.

[17] Migo, E.M., Corbett, K., Graham, J., Smith, S., Tate, S., Moran, P.M. et al. (2006) A novel test of conditioned inhibition correlates with personality measures of schizotypy and reward sensitivity. Behavioural Brain Research, 168, 299-306. doi:10.1016/j.bbr.2005.11.021

[18] Langleben, D.D., Monterosso, J., Elman, I., Ash, B., Krikorian, G. and Austin, G. (2006) Effect of methylphenidate on Stroop color-word task performance in children with attention deficit hyperactivity disorder. Psychiatry Research, 141, 315-320. doi:10.1016/j.psychres.2005.09.007

[19] Lopez, F., Silva, R., Pestreich, L. and Muniz, R. (2003) Comparative efficacy of two once daily methylphenidate formulations (Ritalin LA and Concerta) and placebo in children with attention deficit hyperactivity disorder across the school day. Pediatric Drugs, 5, 545-555. doi:10.2165/00148581-200305080-00005

[20] Harmer, C.J. and Phillips, G.D. (1999) Enhanced conditioned inhibition following repeated pretreatment with d-amphetamine. Psychopharmacology, 142, 120-131. doi:10.1007/s002130050870

[21] Batty, M.J., Liddle, E.B., Pitiot, A., Toro, R., Groom, M.J., Scerif, G., et al. (2010) Cortical gray matter in attention-deficit/hyperactivity disorder: A structural magnetic resonance imaging study. Journal of the American Academy of Child and Adolescent Psychiatry, 49, 229-238.

[22] Groom, M.J., Gaia, S., Liddle, P.F., Batty, M.J., Liddle, E.B., Roberts, K.L., et al. (2010) Effects of motivation and medication on electrophysiological markers of response inhibition in children with attention deficithyperactivity disorder. Biological Psychiatry, 67, 624-631. doi:10.1016/i.biopsych.2009.09.029

[23] Goodman, R., Ford, T., Richards, H., Gatward, R. and Meltzer, H. (2000) The development and well-being assessment: Description and initial validation of an integrated assessment of child and adolescent psychopathology. Journal of Child Psychology and Psychiatry and Allied Disciplines, 41, 645-655. doi:10.1111/j.1469-7610.2000.tb02345.x

[24] Goodman, R. (1997) The strengths and difficulties questionnaire: A research note. Journal of Child Psychology and Psychiatry and Allied Disciplines, 38, 581-586. doi:10.1111/j.1469-7610.1997.tb01545.x

[25] Goodman, R. (2001) Psychometric properties of the strengths and difficulties questionnaire. Journal of the American Academy of Child and Adolescent Psychiatry, 40, 1337-1345. doi:10.1097/00004583-200111000-00015

[26] Conners, C.K. (1997) Conner's rating scales-revised:
Technical manual. Multi-Health Systems, Toronto.

[27] Rutter, M., Bailey, A. and Lord, C. (2003) Social communication questionnaire: Manual. Western Psychological Services, Los Angeles.

[28] Abelson, R.P. (1995) Statistics as principled argument. Lawrence Erlbaum Associates, Hove, 104-131.

[29] Bitsakou, P., Psychogiou, L., Thompson, M. and Sonuga -Barke, E.J.S. (2008) Inhibitory deficits in attention deficit/hyperactivity disorder are independent of basic processing efficiency and IQ. Journal of Neural Transmission, 115, 261-268. doi:10.1007/s00702-007-0828-z

[30] Karazinov, D.M. and Boakes, R.A. (2007) Second-order conditioning in human predictive judgements when there is little time to think. Quarterly Journal of Experimental Psychology, 60, 448-460. doi:10.1080/17470210601002488

[31] Mitchell, C.J., Livesey, E. and Lovibond, P.F. (2007) A dissociation between causal judgement and the ease with which a cause is categorized with its effect. Quarterly Journal of Experimental Psychology, 60, 400-417. doi:10.1080/17470210601002512

[32] Holland, P.C. (1984) Differential effects of reinforcement of an inhibitory feature after serial and simultaneous feature negative discrimination training. Journal of Experimental Psychology: Animal Behavior Processes, 10, 461-475. doi:10.1037/0097-7403.10.4.461

[33] Holland, P.C. and Lamarre, J. (1984) Transfer of inhibition after serial and simultaneous feature negative discrimination training. Learning and Motivation, 15, 219243. doi:10.1016/0023-9690(84)90020-1

[34] Horsley, R.R. and Cassaday, H.J. (2007) Methylphenidate can reduce selectivity in associative learning in an aversive trace conditioning task. Journal of Psychopharmacology, 21, 492-500.

[35] Cassaday, H.J., Finger, B.C. and Horsley, R.R. (2008) Methylphenidate and nicotine focus responding to an informative discrete CS over successive sessions of appetitive conditioning. Journal of Psychopharmacology, 22, 849-859. doi:10.1177/0269881107083842

[36] Lansbergen, M.M., Kenemans, J.L. and van Engeland, H. (2007) Stroop interference and attention-deficit/hyperactivity disorder: A review and meta-analysis. Neuropsychology, 21, 251-262. doi:10.1037/0894-4105.21.2.251

[37] Scheres, A., Oosterlaan, J., Swanson, J., Morein-Zamir, S., Meiran, N., Schut, H., et al. (2003) The effect of methylphenidate on three forms of response inhibition in boys with AD/HD. Journal of Abnormal Child Psychology, 31, 105-120. doi:10.1023/A:1021729501230 